\title{
Phenylpropanoids inhibit protofilament formation of Escherichia coli cell division protein FtsZ
}

\author{
Shanmugam Hemaiswarya, Rohini Soudaminikkutty, \\ Mohana Lakshmi Narasumani and Mukesh Doble
}

Correspondence

Mukesh Doble

mukeshd@iitm.ac.in

Received 19 January 2011

Accepted 1 April 2011

\author{
Department of Biotechnology, Indian Institute of Technology Madras, Chennai 600 036, India
}

\section{INTRODUCTION}

Antimicrobial resistance is a global concern due to its increased prevalence. The major issues are the reduced susceptibility to currently available antimicrobial agents and shortage of newly approved compounds. A growing number of Gram-positive (Staphylococcus aureus, Streptococcus pneumoniae, etc.) and Gram-negative (Pseudomonas aeruginosa, Klebsiella pneumoniae, Escherichia coli, etc.) pathogens are responsible for infection in both the community and health-care settings (Furtado \& Nicolau, 2010). Around 19$60 \%$ of Staphylococcus aureus isolates from ocular infections have been shown to be resistant to macrolides, penicillin and fluoroquinolones (McDonald \& Blondeau, 2010). The overall levels of antibiotic resistance can be dramatically decreased by focusing on the overuse of antibiotics, the pharmacokinetic and pharmacodynamic properties of different drug formulations, and use of proper hygiene

Abbreviations: CD, circular dichroism; OSAR, quantitative structureactivity relationship.

Figures showing phenylpropanoid-induced filament formation in $B$. subtilis 168, the interaction of $E$. coli FtsZ with chlorogenic acid and a contribution plot of hydrophobic interactions are available as supplementary data with the online version of this paper. and protective barriers. There is also a need for the introduction of new antimicrobial agents with novel targets.

FtsZ is a bacterial cytoskeleton protein which assembles into a protofilament in a GTP-dependent manner. This forms a dynamic Z-ring at the mid-cell position. A study done in E. coli showed that 12 different proteins congregate at the Z-ring in a sequentially dependent pathway, causing assembly of the septal ring that guides the synthesis of the circumferential septum (Buddelmeijer \& Beckwith, 2002; Bernhardt \& de Boer, 2003; Romberg \& Levin, 2003; Schmidt et al., 2004). The septal ring constricts in concert with septal progression, culminating in complete disassembly as soon as it matures. Inhibition of this central protein perturbs the formation of the Z-ring and bacterial cytokinesis (Margolin, 2005). FtsZ has become an attractive target, due to its evolutionary distance from eukaryotic tubulin. There are many efforts to identify inhibitors of FtsZ that do not target eukaryotic tubulin. Interestingly, most of the agents that target tubulin/microtubule, including paclitaxel, vinblastine and colchicine, do not affect the dynamics of FtsZ assembly (Jaiswal et al., 2007), indicating that the latter can be a selective antibacterial target.

PC190723, a synthetic compound (substituted benzamide and thiazolopyridine moieties linked with an ether bond), has been found to kill meticillin-resistant Staphylococcus 
aureus, many species of Staphylococcus, Streptococcus species, Bacillus subtilis and Gram-negative bacteria by targeting FtsZ (Haydon et al., 2008). A range of compounds from natural sources including cinnamaldehyde (Domadia et al., 2007), berberine (Domadia et al., 2008), curcumin (Rai et al., 2008), sanguinarine (Beuria et al., 2005), ( \pm )-dichamanetin and ( \pm )-2"' -hydroxy-5"'-benzylisouvarinol-B (Urgaonkar et al., 2005) have been reported to inhibit E. coli FtsZ. Small molecule inhibitors including 2-alkoxycarbonylaminopyridines (White et al., 2002) and totarol (Jaiswal et al., 2007) perturbed the functional properties of FtsZ from Mycobacterium tuberculosis.

The current study exploits the class of phenylpropanoids as FtsZ inhibitors. These compounds make up the majority of polyphenols consumed in our diet (Korkina, 2007). Members belonging to this class such as cinnamic acid, $p$ coumaric acid, caffeic acid, chlorogenic acid (PuupponenPimiä et al., 2001), eugenol (Braga et al., 2007; Hemaiswarya \& Doble, 2009) and ferulic acid (Naz et al., 2006; Hemaiswarya \& Doble, 2010) acid have been shown to possess antimicrobial activity. The activity of these compounds toward bacteria is attributed to their membranedamaging nature (Sung \& Lee, 2010). They perturb the membrane lipid bilayers, causing the leakage of ions and other materials, as well as form pores and dissipate the electrical potential of the membrane (Sung \& Lee, 2010).

\section{METHODS}

Materials. GTP, 2,4,5-trimethoxycinnamic acid and eugenol were purchased from Sigma. Cinnamic, p-coumaric, caffeic and 3,4dimethoxycinnamic acids, Luria-Bertani medium, Tris/ $\mathrm{HCl}, \mathrm{KCl}$ and $\mathrm{MgCl}_{2}$ were purchased from Himedia. Chlorogenic acid, ferulic acid, MES and IPTG were purchased from SRL. All other chemicals were of analytical grade.

Expression and purification of E. coli FtsZ. E. coli FtsZ (EcFtsZ) was overexpressed and purified from the recombinant E. coli BL21 strain carrying pET11b (kindly provided by Professor H. P. Erickson, Duke University) as described previously (Lu et al., 2001; Romberg et al., 2001). The purity of the protein was analysed by $10 \%$ SDSPAGE and its concentration was determined by the Bradford method (Bradford, 1976). The protein was aliquoted and stored at $-80{ }^{\circ} \mathrm{C}$. FtsZ was put through a cycle of calcium-aided assembly and disassembly to select the active and nonaggregated protein (Romberg et al., 2001). All the following experiments were repeated in triplicate.

The inhibition of FtsZ GTPase activity was measured using a malachite green-phosphomolybdate assay (Akiyama et al., 1996) with $6 \mu \mathrm{M} \mathrm{EcFtsZ} \mathrm{in} \mathrm{HEPES} \mathrm{assembly} \mathrm{buffer} \mathrm{[50} \mathrm{mM} \mathrm{4-(2-hydroxyethyl)-}$ 1-piperazineethanesulfonic acid, $\mathrm{pH} 7.7 ; 5 \mathrm{mM}$ magnesium acetate; $350 \mathrm{mM}$ potassium acetate; $10 \mathrm{mM} \mathrm{CaCl}$ ] with $1 \mathrm{mM}$ GTP. The compounds (in DMSO) were added to achieve a final concentration of $100 \mu \mathrm{M}$ and the DMSO concentration was maintained at $1 \%$. FtsZ was preincubated with or without the phenylpropanoids for $5 \mathrm{~min}$ and the reactions were initiated by adding $1 \mathrm{mM}$ GTP. Aliquots were withdrawn after $15 \mathrm{~min}$ of incubation and quenched with $20 \mathrm{mM}$ EDTA. To $5 \mu \mathrm{l}$ reaction aliquots, $120 \mu \mathrm{l}$ malachite green solution and $30 \mu \mathrm{l} 34 \%(\mathrm{w} / \mathrm{v})$ sodium citrate were added, the samples were incubated for $30 \mathrm{~min}$ at room temperature, and the $A_{650}$ was read
(UV-visible spectrometer V-550; Jasco). The per cent inhibition was calculated by comparing the rate of hydrolysis in the presence and absence of inhibitor.

Inhibition of polymerization ability. Inhibition of GTP-initiated in vitro polymerization of recombinant wild-type E. coli FtsZ by phenylpropanoids was assessed by a light-scattering assay (Mukherjee \& Lutkenhaus, 1999). FtsZ $(6 \mu \mathrm{M})$ was polymerized in HEPES assembly buffer with $1 \mathrm{mM}$ GTP in the presence of phenylpropanoids $(0-250 \mu \mathrm{M})$ and monitored in a Jasco fluorescence spectrometer with excitation and emission wavelengths set at $350 \mathrm{~nm}$. Data were plotted as per cent inhibition of polymerization activity as a function of drug concentration. The curve was fitted to a fourparameter logistic function using SIGMAPLOT 10.0 (Systat Software) and the drug concentration required to inhibit the polymerization ability of FtsZ by $50 \%\left(\mathrm{IC}_{50}\right)$ was estimated.

Visualization of bacterial morphology. B. subtilis 168 was grown in the absence and presence of different concentrations of phenylpropanoids for $2 \mathrm{~h}$ in LB broth supplemented with $10 \mathrm{mM}$ phenylpropanoids. The cells were fixed in $2.8 \%$ formaldehyde and $0.04 \%$ glutaraldehyde at $25{ }^{\circ} \mathrm{C}$. The cells were collected, washed and resuspended in $\mathrm{PBS}$ ( $\mathrm{pH}$ 7.4). Their morphology was examined under a differential interference contrast microscope (Nikon Eclipse 80i) at $\times 40$ magnification. The cell length was measured using ImageJ $1.43 \mathrm{u}$ software (National Institutes of Health, USA).

Electron microscopic analysis. FtsZ at $6 \mu \mathrm{M}$ was polymerized in assembly buffer for $10 \mathrm{~min}$. The protofilaments were treated with 100 $\mu \mathrm{M}$ chlorogenic acid and samples were prepared for electron microscopy (Santra et al., 2005). The images were collected using a JEOL 3010 transmission electron microscope.

Analysis of changes in secondary structure. FtsZ $(6 \mu \mathrm{M})$ was incubated without or with $100 \mu \mathrm{M}$ chlorogenic acid in $25 \mathrm{mM}$ phosphate buffer ( $\mathrm{pH} \mathrm{6.5)}$ for $15 \mathrm{~min}$ at $25^{\circ} \mathrm{C}$. The far-UV circular dichroism (CD) spectrum was monitored over a wavelength range of 190-250 nm using a Jasco J720 dichrograph equipped with a Peltier temperature controller and a $0.1 \mathrm{~cm}$ path length quartz cuvette. A mean of five scans was taken for each spectrum. K2D2, a method with an associated web server, was used to estimate protein secondary structure from CD spectra (Perez-Iratxeta \& Andrade-Navarro, 2008).

Molecular modelling. The $3 \mathrm{D}$ structure of E. coli cell division protein FtsZ was constructed from the crystal structure of Methanococcus jannaschii FtsZ (PDB code: 1FSZ, $2.8 \AA$ ) (Domadia et al., 2007; Löwe \& Amos, 1998). Homology modelling was performed by using MODELLER version 9v7 (Šali \& Blundell, 1993). The structures of the ligands were energy minimized and charges were added using ANTECHAMBER (AM1-BCC). The energy minimized ligands were docked to the modelled protein with AutoDock version 3.0 (Morris et al., 1998). The grid maps for docking were prepared with Autogrid3. The active site was selected as the centre of the grid box and $56 \times 46 \times 52$ points with grid spacing of $0.375 \AA$ were calculated. A Lamarckian genetic algorithm was adopted to perform the molecular docking. The various genetic algorithm parameters were: a maximum of 250000 energy evaluations; a maximum of 27000 generations; mutation and crossover rates of 0.02 and 0.8 , respectively; and 100 docking runs. The final docked conformations were clustered with a tolerance of $1.5 \AA$ root-mean-square deviation. The best docked representation of the ligand was chosen based on the conformation with lowest binding free energy. Hydrogen bonding and hydrophobic interactions of the ligand with the protein were analysed using PyMOL software, v. 0.99 (DeLano, 2002).

Development of a quantitative structure-activity relationship (QSAR). A 3D-quantitative structure-activity relationship (QSAR) 
was generated using V-Life Molecular Design Suite 3.0 (Vlife MDS). The set of eight molecules was divided into training and test sets (six in the training and two in the test sets). Here the activity is defined as $\log [p /(100-p)]$, where $p$ is the percentage inhibition of GTP-initiated polymerization caused by the compounds at a concentration of 100 $\mu \mathrm{M}$. The structures of all the eight compounds were built with the software and their minimum energy conformations were estimated with the Monte Carlo conformational search algorithm with a rootmean-square gradient of $0.001 \mathrm{kcal} \mathrm{mol}^{-1}$ and iteration limit of 10000 using Merck molecular force field 94. All the molecules were aligned on a common template. The steric, electrostatic and hydrophobic interaction energies at grid positions around these aligned molecules were estimated. Steric and electrostatic fields were computed at each grid point by calculating the interaction energy between the molecule and a probe molecule placed at each grid point considering Merck molecular force field charges (Halgren, 1996). The probe consisted of a methyl group of charge +1 with $10.0 \mathrm{kcal} \mathrm{mol}^{-1}$ electrostatic and $30.0 \mathrm{kcal} \mathrm{mol}^{-1}$ steric cut-off. A value of 1.0 is assigned for distance-dependent dielectric constant (Cramer et al., 1988). Several regression relations between these descriptors and the activity were generated using molecular field analysis coupled with partial least-squares. The best model was selected on the basis of various statistical parameters, namely squared correlation coefficient $\left(r^{2}\right)$, cross validated square correlation coefficient $\left(q^{2}\right)$, predicted $r^{2}$ and sequential Fisher test (F).

\section{RESULTS}

\section{Phenylpropanoids inhibit GTPase activity}

The dynamics of FtsZ assembly is regulated by the hydrolysis of GTP (Mukherjee et al., 2001). Most of the phenylpropanoids reduced the GTPase activity of $E c \mathrm{FtsZ}$ at a concentration of $100 \mu \mathrm{M}$ (Fig. 1). For example, $100 \mu \mathrm{M}$ chlorogenic and ferulic acids inhibited the GTPase activity by 46 and $34 \%$, respectively. Caffeic and p-coumaric acid reduced GTPase activity by 23 and $29 \%$, respectively, when compared to the control $(P<0.001)$. The rest of the phenylpropanoids exhibited lesser activity (in the range of $15-20 \%$ ) than the above compounds. GTPase activity is required for the dynamics of FtsZ assembly and therefore inhibition of this activity will perturb the formation of protofilament.

\section{Inhibition of FtsZ assembly by phenylpropanoids}

The kinetics of assembly of FtsZ is measured semiquantitatively in vitro in terms of an increase in the light scattering at $350 \mathrm{~nm}$ (Mukherjee \& Lutkenhaus, 1999). Most of the phenylpropanoids inhibited FtsZ assembly in a dose-dependent manner (Fig. 2). Chlorogenic acid inhibited GTP-initiated FtsZ polymerization by 77, 57, 45 and $27 \%$ at $200,100,50$ and $25 \mu \mathrm{M}$ concentrations, respectively, after $15 \mathrm{~min}$ and the $\mathrm{IC}_{50}$ was estimated to be approximately $70 \mu \mathrm{M}$. Table 1 lists the effect of phenylpropanoids on the polymerization of EcFtsZ. Caffeic, p-coumaric and 2,4,5-trimethoxycinnamic acids inhibited FtsZ assembly with $\mathrm{IC}_{50}$ values between 100 and $200 \mu \mathrm{M}$. The $\mathrm{IC}_{50}$ values for eugenol and ferulic and 3,4dimethoxycinnamic acids were more than $250 \mu \mathrm{M}$. The effect of chlorogenic acid on the assembly of FtsZ was visualized by transmission electron microscopy (Fig. 3). The control showed thick bundles of FtsZ polymers. A high concentration $(100 \mu \mathrm{M})$ of chlorogenic acid under the polymerization conditions induced aggregation of FtsZ monomers and distorted protofilaments.

\section{Effect of phenylpropanoids on the morphology of B. subtilis 168}

Most of the phenylpropanoids induced filamentation in $B$. subtilis 168 cells. The mean length of this micro-organism was $5.4 \pm 1.3 \mu \mathrm{m}$ and it increased by 3.2 -fold to $13.7 \pm 6.2$ $\mu \mathrm{m}$ after $2 \mathrm{~h}$ of incubation with $100 \mathrm{mM}$ chlorogenic acid. In the presence of $100 \mathrm{mM}$ cinnamic, $p$-coumaric, caffeic, ferulic and 2,4,5-trimethoxycinnamic acids, the mean length of B. subtilis 168 cells increased by one- to twofold

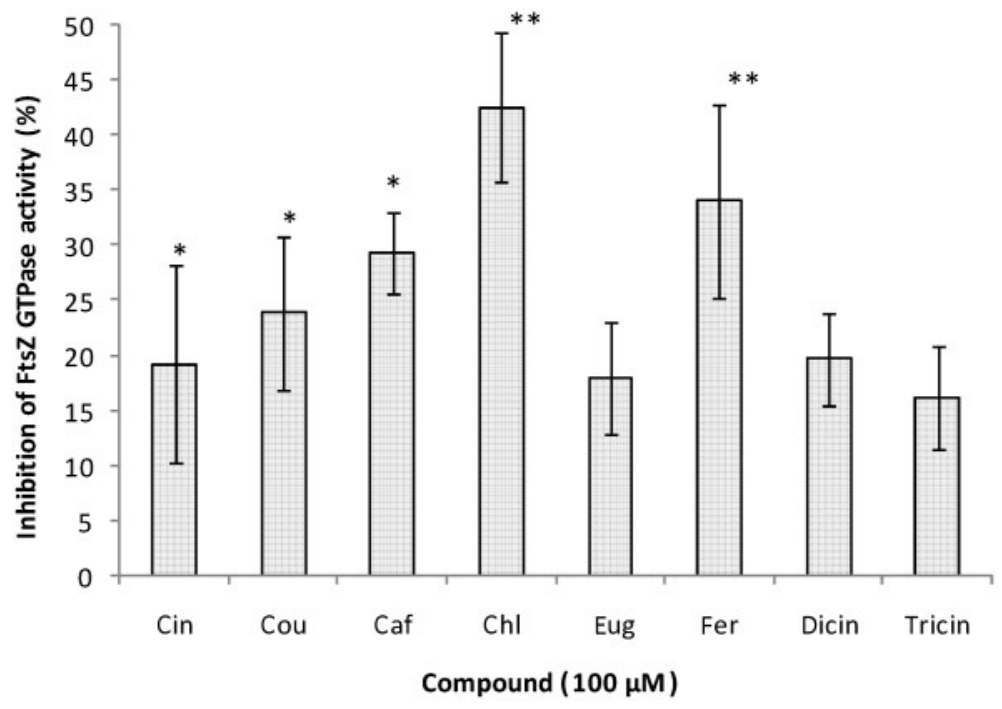

Fig. 1. Effect of phenylpropanoids on the GTPase activity (15 min) of E. coli FtsZ. ${ }^{\star \star} P<0.001 ;{ }^{\star} P<0.05$. 


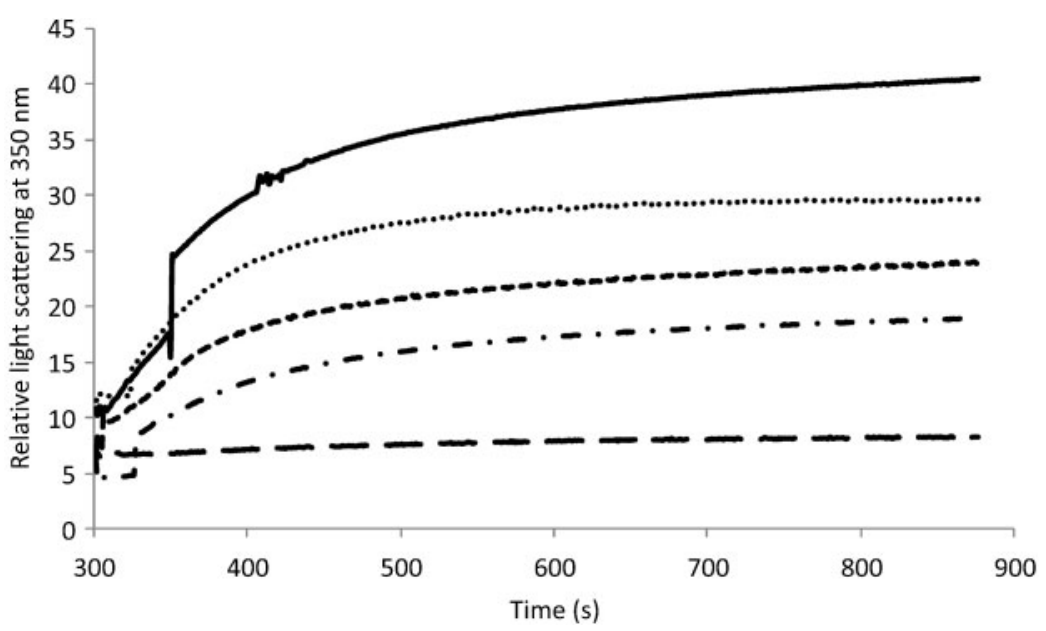

Fig. 2. FtsZ polymerized in the absence (-) and presence of $25 \mu \mathrm{M}(\ldots), 50 \mu \mathrm{M}(--)$, $100 \mu \mathrm{M}(-.-)$ and $200 \mu \mathrm{M}(--)$ chlorogenic acid.
(Table 2). Eugenol and 3,4-dimethoxycinnamic acid did not increase the mean length.

Most of the phenylpropanoid-treated cells were strikingly longer than the control (vehicle-treated) cells. In the case of the control, $44 \%$ of the cells had a mean length in the range of $3-5 \mu \mathrm{m}$ (Supplementary Fig. S1 in JMM Online). More than $40 \%$ of cells treated with caffeic, chlorogenic, ferulic and 2,4,5-trimethoxycinnamic acids had a length $>11 \mu \mathrm{m}$ and more than $30 \%$ of cells were $>11 \mu \mathrm{m}$ when treated with cinnamic and $p$-coumaric acid. These results indicate that phenylpropanoids inhibit bacterial proliferation by inhibiting cytokinesis and thereby increase the cell length.

\section{Effect of chlorogenic acid on the secondary structure of FtsZ}

The effect of $100 \mu \mathrm{M}$ chlorogenic acid on the secondary structure of FtsZ was determined with far-UV CD spectra. Analysis of these spectra using deconvolution software, K2D2 (Perez-Iratxeta \& Andrade-Navarro, 2008), indicated that the secondary structure of FtsZ contained $39.37 \% \pm 0.8$ and $11.52 \% \pm 0.6$ of $\alpha$-helix and $\beta$-strand, respectively. Chlorogenic acid perturbed the secondary structure of FtsZ (Fig. 4) by reducing the helical content by $23.8 \%$ (from $39.37 \%$ to $29.97 \pm 0.5 \%$ ) and increasing the $\beta$-strand content by $18.6 \%$ (from $11.52 \%$ to $13.66 \%$ ). The troughs at 208 and $222 \mathrm{~nm}$ are characteristic of the high content of $\alpha$-helix, which is disrupted due to chlorogenic acid treatment. These observed changes were statistically significant $(P<0.001)$.

\section{Modelling}

The amino acids in the active sites of E. coli cell division protein FtsZ are Asn207, Asp209, Asp212 and Arg214, which are equivalent to those found in $M$. jannaschii (namely Asn233, Asp235, Asp238 and Lys240). These two proteins share a sequence similarity of $46.3 \%$. Hence the structure of M. jannaschii FtsZ (PDB code: 1FSZ) can be considered a valid template for building the homology model. Docking of various compounds to the protein showed that chlorogenic acid had the highest binding energy when compared to the rest (Table 3). Hydroxyl groups in chlorogenic acid formed hydrogen bonds with Ala11, Gly36, Asn207, Val208, Asp209 and Phe210 (Supplementary Fig. S2). The complex was further stabilized by the hydrophobic interaction with Pro203 and polar contact with Asn207. The latter is located in the T7 loop of the protein and it has been suggested to be part of the active site region (Scheffers et al., 2002). The order of increase in binding energy was chlorogenic acid $>$ ferulic acid $>$ caffeic acid $>p$-coumaric acid $>$ cinnamic acid $>3,4$ dimethoxycinnamic acid $>$ eugenol $>2,4,5$-trimethoxycinnamic acid. Almost all the phenylpropanoids interacted with at least one residue in the T7 loop.

\section{QSAR analysis}

The inhibition of GTPase activity of FtsZ by phenylpropanoids at a concentration of $100 \mu \mathrm{M}$ was used to generate the 3D-QSAR. The percentage inhibition reported in Table 1 was converted to activity, defined as $\log [p /(100-p)]$ : activity $=-(1.8)$ H_214+0.01, $r^{2}=0.7, q^{2}=0.6, \quad \mathrm{~F}=10.6$, pred_r ${ }^{2}=0.6$.

H_214 is a hydrophobic descriptor at the spatial grid point 214 (Supplementary Fig. S3). A hydrophobic descriptor with negative coefficient (H_214) around the third and fourth position of the benzene ring indicates that hydrophilic groups are preferred for enhanced activity. All the statistical parameters estimated were reasonably good indicating the quality of the QSAR developed.

\section{DISCUSSION}

Phenylpropanoids belong to the largest group of secondary metabolites produced by plants, mainly in response to biotic or abiotic stresses such as infections, wounding, UV irradiation, pollutants and other hostile environmental 
Table 1. Effect of phenylpropanoids on in vitro assembly of FtsZ

The polymerization of GTP-induced FtsZ was monitored as a function of light scattering for 15 min.

\begin{tabular}{|c|c|c|c|}
\hline Compound & Structure & $\mathrm{IC}_{50}(\mu \mathrm{M}) \pm \mathrm{SEM}$ & $\%$ inhibition $(100 \mu \mathrm{M})$ \\
\hline$p$-Coumaric acid & & $189.53 \pm 3.7$ & $26.83 \pm 3.1$ \\
\hline Caffeic acid & & $105.96 \pm 6.3$ & $45.76 \pm 12.9$ \\
\hline Chlorogenic acid & & $69.55 \pm 3.6$ & $57.04 \pm 5.6$ \\
\hline Eugenol & & $>250$ & $15.89 \pm 2.6$ \\
\hline Ferulic acid & & $>250$ & $19.1 \pm 2.3$ \\
\hline 3,4-Dimethoxycinnamic acid & & $>250$ & $12.7 \pm 3.5$ \\
\hline 2,4,5-Trimethoxycinnamic acid & & $148.59 \pm 4.3$ & $29.8 \pm 5.2$ \\
\hline
\end{tabular}

conditions (Korkina, 2007). The current study shows that they inhibit FtsZ assembly, thereby perturbing the formation of the Z-ring, and finally inhibiting the process of cell division. This inhibition was confirmed by the reduction in light scattering of polymerized FtsZ. They also inhibited GTPase activity of FtsZ, indicating their ability to depolymerize preformed FtsZ polymers. Structures similar to the phenylpropanoids such as curcumin (a dimer with a phenylpropanoid group) and cinnamaldehyde have been reported to inhibit E. coli FtsZ (Domadia et al., 2007; Rai et al., 2008).
In the current list of eight phenylpropanoids, chlorogenic $\left(\mathrm{IC}_{50}=70 \mu \mathrm{M}\right)$ and caffeic acids were good inhibitors of FtsZ. Chlorogenic acid is an ester of caffeic acid and quinic acid. The decrease in the polymerization and bundling of FtsZ may be due to the conformational changes induced by chlorogenic acid in the FtsZ monomer. FtsZ may not be the primary target for chlorogenic acid. Antifungal studies against Candida albicans indicated that it disrupts the structure of the cell membrane (Sung \& Lee, 2010). Caffeic acid also inhibited FtsZ assembly, though at a slightly 


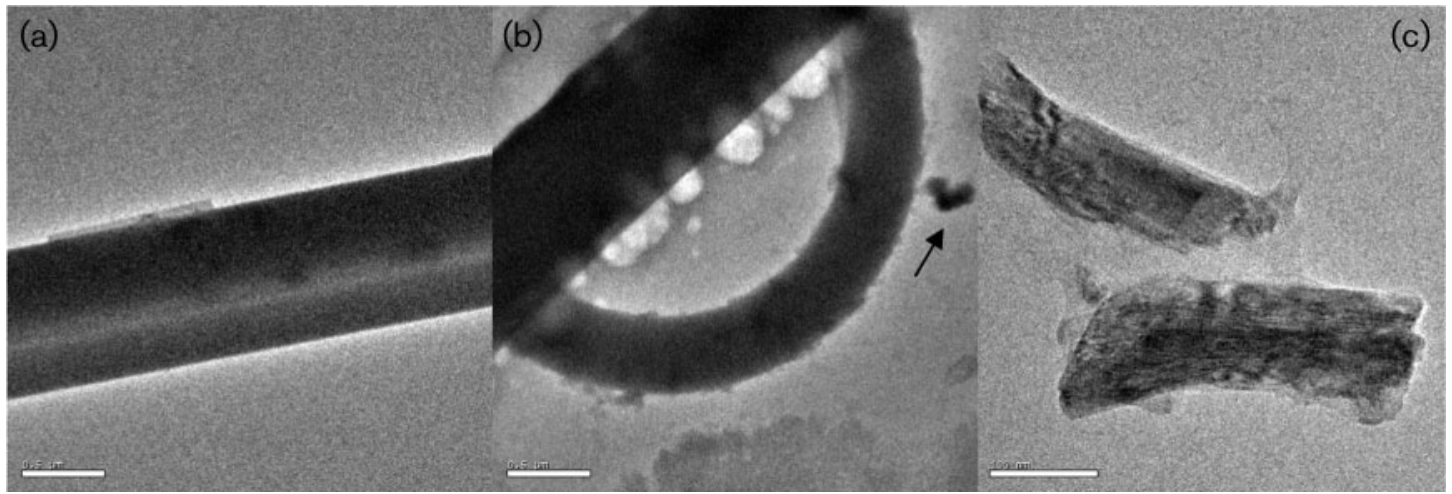

Fig. 3. Inhibitory effect of chlorogenic acid on preformed FtsZ wild-type protofilaments as viewed by electron microscopy. (a) Control; (b, c) treated with $100 \mu \mathrm{M}$ chlorogenic acid. The arrow indicates aggregates formed. (c) Distorted protofilaments. Bars, $500 \mu \mathrm{m}(\mathrm{a}, \mathrm{b})$ and $100 \mu \mathrm{m}(\mathrm{c})$.

higher $\mathrm{IC}_{50}($ at $106 \mu \mathrm{M}$ ), followed by $p$-coumaric acid. Caffeic and $p$-coumaric acid were reported by other researchers to cause 44 and $59 \%$ membrane damage, respectively, on a Gram-positive bacterium, Oenococcus oeni (Campos et al., 2009). This suggests that these phenylpropanoids act not only through targeting FtsZ but also through membrane perturbation. Ferulic acid at $100 \mu \mathrm{M}$ has high GTPase inhibition activity ( $26 \%)$. Though the inhibition of polymerization ability of FtsZ by ferulic acid was poor ( $\mathrm{IC}_{50}$ $>250 \mu \mathrm{M}$ ), there was a significant increase in the cell length of B. subtilis 168 . Also ferulic acid at $10 \mathrm{mM}$ has been shown to possess a very low membrane-damaging activity (less than $5 \%$ ) against Gram-negative bacteria (Hemaiswarya \& Doble, 2010). The phenylpropanoids being small molecules have multiple targets. Eugenol was found to be less active against the inhibition of GTPase and polymerization activities. There was no increase in the cell size of B. subtilis 168 treated with eugenol when compared with that of the control. Eugenol is reported to primarily act by disrupting the cytoplasmic membrane (Hemaiswarya \& Doble, 2009; Gill \& Holley, 2006). Cinnamic, p-coumaric, caffeic, chlorogenic and 2,4,

Table 2. Mean length of $B$. subtilis 168 cells in the presence $(100 \mu \mathrm{M})$ and absence of phenylpropanoids

\begin{tabular}{|lc|}
\hline Treatment & Mean length $(\boldsymbol{\mu m})$ \\
\hline Control (without treatment) & $5.4 \pm 1.3$ \\
Cinnamic acid & $12.7 \pm 8.9^{\star}$ \\
p-Coumaric acid & $11.9 \pm 8.4^{\star}$ \\
Caffeic acid & $11.9 \pm 8.2^{*}$ \\
Chlorogenic acid & $13.67 \pm 6.2^{\star}$ \\
Eugenol & $5.24 \pm 3.9$ \\
Ferulic acid & $10.9 \pm 9.3^{\star}$ \\
3,4-Dimethoxycinnamic acid & $6.9 \pm 4.4^{\star}$ \\
2,4,5-Trimethoxycinnamic acid & $12.06 \pm 8.9^{\star}$ \\
\hline
\end{tabular}

${ }^{\star} P<0.05$. 5-trimethoxycinnamic acids and eugenol have been shown to damage the bacterial membrane of Gram-negative and Gram-positive bacteria (Hemaiswarya \& Doble, 2009; Gill \& Holley, 2006). Previous studies (Rastogi et al., 2008) have shown the potential of a few phytochemicals to inhibit FtsZ assembly under in vitro conditions. trans-Cinnamic acid was found to be the most potent inhibitor; however, naringenin, eugenol and 4-formyl cinnamic acid did not show considerable inhibition of FtsZ polymerization. Our results also substantiate the above study, where cinnamic acid inhibited FtsZ assembly and eugenol was inactive.

Docking studies indicated that all the phenylpropanoids interacted with at least one of the residues in the T7 loop of FtsZ. Structural analysis of the organization of FtsZ subunits in the polymers, in wild-types as well in mutants, indicates the important role of the T7-loop region ('tubulin-loop' no. 7) (Nogales et al., 1998) of FtsZ in the self-association of the monomers. The T7 loop is highly conserved among FtsZ proteins from different species. Modelling of the crystal structure of M. jannaschii FtsZ1 onto electron microscopy images of protofilaments of FtsZ1 has shown that the active site for GTP hydrolysis may be shared by two FtsZ subunits, with the GTP-binding domain located on one monomer and modulation of hydrolysis by the T7 loop of the other domain (Löwe \& Amos, 1999). FtsZ proteins contain a conserved sequence that is homologous to the Walker B motif present in a large number of ATP-binding proteins (Walker et al., 1982; Burke et al., 1990). The E. coli FtsZ residues 208-212 (VDFAD) conform to the motif '(hydrophobic, aliphatic)$\mathrm{X}$-aromatic-X-acidic' (part of the Walker $\mathrm{B}$ sequence), which has been implicated as the $\mathrm{Mg}^{2+}$-binding site consensus sequence in adenylate kinase, ATPases and myosins (Walker et al., 1982; Burke et al., 1990; Farr \& Sternlicht, 1992). Interaction of phenylpropanoids with these residues would probably result in the perturbation of the conformation of the T7 loop, which is involved in the GTPase activity of the FtsZ (Scheffers et al., 2002). Our 


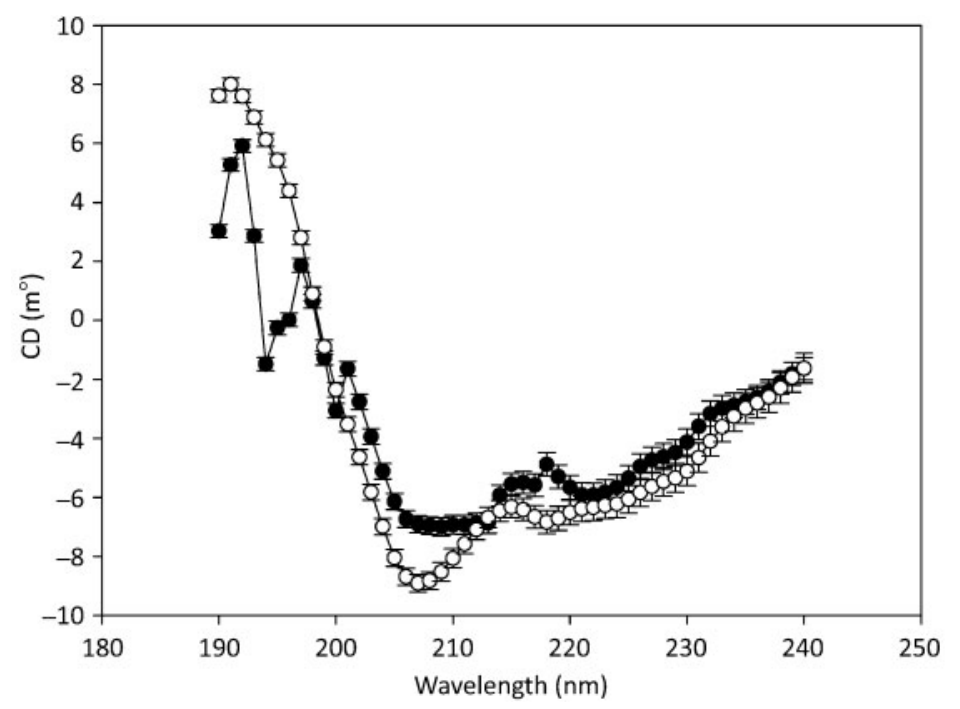

Fig. 4. CD spectra of FtsZ alone $(\bigcirc)$ and treated with $100 \mu \mathrm{M}$ chlorogenic acid $(\bullet)$.

experiments have shown that phenylpropanoids inhibit the GTPase activity of FtsZ, further validating the above proposition. The crystal structure of the SOS-inducible cell division inhibitor SulA in complex with FtsZ from Pseudomonas aeruginosa revealed that SulA binds to the T7-loop surface of FtsZ (Cordell et al., 2003). These compounds may bind to the T7 loop in one FtsZ monomer, inducing such conformational changes that it fails to make optimum contact with the GTP binding

Table 3. Docking results with modelled FtsZ (E. coli)

\begin{tabular}{|c|c|c|c|}
\hline Ligand & $\begin{array}{c}\text { Binding } \\
\text { energy }\end{array}$ & H-bonds & $\begin{array}{c}\text { Distance } \\
(\AA)\end{array}$ \\
\hline \multirow[t]{2}{*}{ Cinnamic acid } & \multirow[t]{2}{*}{-5.68} & Met206 & 2.158 \\
\hline & & Thr296 & 1.898 \\
\hline \multirow[t]{2}{*}{$p$-Coumaric acid } & \multirow[t]{2}{*}{-5.72} & Met206 & 2.219 \\
\hline & & Thr296 & 1.866 \\
\hline \multirow[t]{2}{*}{ Caffeic acid } & \multirow[t]{2}{*}{-6.17} & Met206 & 2.212 \\
\hline & & Thr296 & 1.849 \\
\hline \multirow[t]{6}{*}{ Chlorogenic acid } & \multirow[t]{6}{*}{-8.36} & Ala11 & 1.777 \\
\hline & & Gly36 & 1.949 \\
\hline & & Asn 207 & 2.12 \\
\hline & & Val208 & 2.217 \\
\hline & & Asp209 & 2.138 \\
\hline & & Phe210 & 2.168 \\
\hline Eugenol & -5.26 & Thr201 & 2.228 \\
\hline \multirow[t]{4}{*}{ Ferulic acid } & \multirow[t]{4}{*}{-6.33} & Ala11 & 2.194 \\
\hline & & Gly204 & 2.107 \\
\hline & & Met206 & 1.783 \\
\hline & & Val208 & 2.236 \\
\hline \multirow[t]{2}{*}{ 3,4-Dimethoxycinnamic acid } & \multirow[t]{2}{*}{-5.46} & Ala11 & 1.834 \\
\hline & & Val208 & 1.691 \\
\hline \multirow[t]{2}{*}{ 2,4,5-Trimethoxycinnamic acid } & \multirow[t]{2}{*}{-5.18} & Ala11 & 2.079 \\
\hline & & Val208 & 1.689 \\
\hline
\end{tabular}

T1-T6 loop in the neighbouring monomer and thereby inhibiting FtsZ polymerization.

The 3D-QSAR indicates the need for hydrophilic groups at the third and fourth carbon of the benzene ring for high activity. Chlorogenic and caffeic acid possess a hydroxyl group, making them more hydrophilic than the other compounds, which have methoxy substituents, hence they exhibit highest activity. The presence of more hydroxyl groups favours hydrogen bonds with the amino acid side chains in the active site. Molecular docking studies also indicate that chlorogenic acid interacts with FtsZ through hydrogen bonds.

The present work suggests that phenylpropanoids could be considered as a new source for the development of antibacterial drugs. The study involves inhibition under in vitro conditions, and their in vivo performance has to be speculated. Chlorogenic and caffeic acids could be the lead structures for the design of more stable and potent antiFtsZ agents as well as a vital tool for understanding the regulatory role of FtsZ in cell division. Cell wall damage, which remains a likely mode of action, is known to induce the SOS response and cell filamentation. Two additional experiments are needed to support the above claims, namely: (i) induction of cell filamentation in an SOS mutant of an E. coli strain, which will control again many stress related pathways to filamentation; and (ii) over- or underexpression of the gene encoding FtsZ, leading to resistance of sensitivity to the compounds, which will help to prove whether the compounds act by FtsZ inhibition or not. Also, it would be worth trying to visualize the Z-rings by fluorescence microscopy and see the effect of these compounds on their formation.

\section{ACKNOWLEDGEMENTS}

The authors thank Professor H. P. Erickson (Duke University) for providing the E. coli BL21 strain carrying pET11b. 


\section{REFERENCES}

Akiyama, Y., Kihara, A., Tokuda, H. \& Ito, K. (1996). FtsH (HflB) is an ATP-dependent protease selectively acting on SecY and some other membrane proteins. J Biol Chem 271, 31196-31201.

Bernhardt, T. G. \& de Boer, P. A. (2003). The Escherichia coli amidase AmiC is a periplasmic septal ring component exported via the twinarginine transport pathway. Mol Microbiol 48, 1171-1182.

Beuria, T. K., Santra, M. K. \& Panda, D. (2005). Sanguinarine blocks cytokinesis in bacteria by inhibiting FtsZ assembly and bundling. Biochemistry 44, 16584-16593.

Bradford, M. M. (1976). A rapid and sensitive method for the quantitation of microgram quantities of protein utilizing the principle of protein-dye binding. Anal Biochem 72, 248-254.

Braga, P. C., Sasso, M. D., Culici, M. \& Alfieri, M. (2007). Eugenol and thymol, alone or in combination, induce morphological alterations in the envelope of Candida albicans. Fitoterapia 78, 396-400.

Buddelmeijer, N. \& Beckwith, J. (2002). Assembly of cell division proteins at the E. coli cell center. Curr Opin Microbiol 5, 553-557.

Burke, M., Rajasekharan, K. N., Maruta, S. \& Ikebe, M. (1990). A second consensus sequence of ATP-requiring proteins resides in the 21-kDa C-terminal segment of myosin subfragment 1. FEBS Lett 262, 185-188.

Campos, F. M., Couto, J. A., Figueiredo, A. R., Tóth, I. V., Rangel, A. O. S. S. \& Hogg, T. A. (2009). Cell membrane damage induced by phenolic acids on wine lactic acid bacteria. Int J Food Microbiol 135, 144-151.

Cordell, S. C., Robinson, E. J. H. \& Lowe, J. (2003). Crystal structure of the SOS cell division inhibitor SulA and in complex with FtsZ. Proc Natl Acad Sci U S A 100, 7889-7894.

Cramer, R. D., Patterson, D. E. \& Bunce, J. D. (1988). Comparative molecular field analysis (CoMFA). 1. Effect of shape on binding of steroids to carrier proteins. J Am Chem Soc 110, 5959-5967.

DeLano, W. L. (2002). The PyMOL Molecular Graphics System. Palo Alto, CA: DeLano Scientific.

Domadia, P., Swarup, S., Bhunia, A., Sivaraman, J. \& Dasgupta, D. (2007). Inhibition of bacterial cell division protein FtsZ by cinnamaldehyde. Biochem Pharmacol 74, 831-840.

Domadia, P. N., Bhunia, A., Sivaraman, J., Swarup, S. \& Dasgupta, D. (2008). Berberine targets assembly of Escherichia coli cell division protein FtsZ. Biochemistry 47, 3225-3234.

Farr, G. W. \& Sternlicht, H. (1992). Site-directed mutagenesis of the GTP-binding domain of $\beta$-tubulin. J Mol Biol 227, 307-321.

Furtado, G. H. \& Nicolau, D. P. (2010). Overview perspective of bacterial resistance. Expert Opin Ther Pat 20, 1273-1276.

Gill, A. O. \& Holley, R. A. (2006). Disruption of Escherichia coli, Listeria monocytogenes and Lactobacillus sakei cellular membranes by plant oil aromatics. Int J Food Microbiol 108, 1-9.

Halgren, T. A. (1996). Merck molecular force field. III. Molecular geometries and vibrational frequencies. J Comput Chem 17, 553-586.

Haydon, D. J., Stokes, N. R., Ure, R., Galbraith, G., Bennett, J. M., Brown, D. R., Baker, P. J., Barynin, V. V., Rice, D. W. \& other authors (2008). An inhibitor of FtsZ with potent and selective antistaphylococcal activity. Science 321, 1673-1675.

Hemaiswarya, S. \& Doble, M. (2009). Synergistic interaction of eugenol with antibiotics against Gram negative bacteria. Phytomedicine 16, 997-1005.

Hemaiswarya, S. \& Doble, M. (2010). Synergistic interaction of phenylpropanoids with antibiotics against bacteria. J Med Microbiol 59, 1469-1476.
Jaiswal, R., Beuria, T. K., Mohan, R., Mahajan, S. K. \& Panda, D. (2007). Totarol inhibits bacterial cytokinesis by perturbing the assembly dynamics of FtsZ. Biochemistry 46, 4211-4220.

Korkina, L. G. (2007). Phenylpropanoids as naturally occurring antioxidants: from plant defense to human health. Cell Mol Biol (Noisy-le-grand) 53, 15-25.

Löwe, J. \& Amos, L. A. (1998). Crystal structure of the bacterial celldivision protein FtsZ. Nature 391, 203-206.

Löwe, J. \& Amos, L. A. (1999). Tubulin-like protofilaments in Ca2+ -induced FtsZ sheets. EMBO J 18, 2364-2371.

Lu, C., Stricker, J. \& Erickson, H. P. (2001). Site-specific mutations of FtsZ - effects on GTPase and in vitro assembly. BMC Microbiol 1, 7.

Margolin, W. (2005). FtsZ and the division of prokaryotic cells and organelles. Nat Rev Mol Cell Biol 6, 862-871.

McDonald, M. \& Blondeau, J. M. (2010). Emerging antibiotic resistance in ocular infections and the role of fluoroquinolones. J Cataract Refract Surg 36, 1588-1598.

Morris, G. M., Goodsell, D. S., Halliday, R. S., Huey, R., Hart, W. E., Belew, R. K. \& Olson, A. J. (1998). Automated docking using a Lamarckian genetic algorithm and empirical binding free energy function. J Comput Chem 19, 1639-1662.

Mukherjee, A. \& Lutkenhaus, J. (1999). Analysis of FtsZ assembly by light scattering and determination of the role of divalent metal cations. J Bacteriol 181, 823-832.

Mukherjee, A., Saez, C. \& Lutkenhaus, J. (2001). Assembly of an FtsZ mutant deficient in GTPase activity has implications for FtsZ assembly and the role of the $\mathrm{Z}$ ring in cell division. J Bacteriol 183, 7190-7197.

Naz, S., Ahmad, S., Ajaz Rasool, S., Asad Sayeed, S. \& Siddiqi, R. (2006). Antibacterial activity directed isolation of compounds from Onosma hispidum. Microbiol Res 161, 43-48.

Nogales, E., Downing, K. H., Amos, L. A. \& Löwe, J. (1998). Tubulin and FtsZ form a distinct family of GTPases. Nat Struct Biol 5, 451-458.

Perez-Iratxeta, C. \& Andrade-Navarro, M. A. (2008). K2D2: estimation of protein secondary structure from circular dichroism spectra. BMC Struct Biol 8, 25.

Puupponen-Pimiä, R., Nohynek, L., Meier, C., Kähkönen, M., Heinonen, M., Hopia, A. \& Oksman-Caldentey, K.-M. (2001). Antimicrobial properties of phenolic compounds from berries. J Appl Microbiol 90, 494-507.

Rai, D., Singh, J. K., Roy, N. \& Panda, D. (2008). Curcumin inhibits FtsZ assembly: an attractive mechanism for its antibacterial activity. Biochem J 410, 147-155.

Rastogi, N., Domadia, P., Shetty, S. \& Dasgupta, D. (2008). Screening of natural phenolic compounds for potential to inhibit bacterial cell division protein FtsZ. Indian J Exp Biol 46, 783-787.

Romberg, L. \& Levin, P. A. (2003). Assembly dynamics of the bacterial cell division protein FtsZ: poised at the edge of stability. Annu Rev Microbiol 57, 125-154.

Romberg, L., Simon, M. \& Erickson, H. P. (2001). Polymerization of FtsZ, a bacterial homolog of tubulin: is assembly cooperative? J Biol Chem 276, 11743-11753.

Šali, A. \& Blundell, T. L. (1993). Comparative protein modelling by satisfaction of spatial restraints. J Mol Biol 234, 779-815.

Santra, M. K., Dasgupta, D. \& Panda, D. (2005). Deuterium oxide promotes assembly and bundling of FtsZ protofilaments. Proteins 61, 1101-1110.

Scheffers, D.-J., de Wit, J. G., den Blaauwen, T. \& Driessen, A. J. M. (2002). GTP hydrolysis of cell division protein FtsZ: evidence that the active site is formed by the association of monomers. Biochemistry $\mathbf{4 1}$, 521-529. 
Schmidt, K. L., Peterson, N. D., Kustusch, R. J., Wissel, M. C., Graham, B., Phillips, G. J. \& Weiss, D. S. (2004). A predicted ABC transporter, FtsEX, is needed for cell division in Escherichia coli. J Bacteriol 186, 785-793.

Sung, W. S. \& Lee, D. G. (2010). Antifungal action of chlorogenic acid against pathogenic fungi, mediated by membrane disruption. Pure Appl Chem 82, 219-226.

Urgaonkar, S., La Pierre, H. S., Meir, I., Lund, H., RayChaudhuri, D. \& Shaw, J. T. (2005). Synthesis of antimicrobial natural products targeting FtsZ: $( \pm)$-dichamanetin and $( \pm)$-2'"'-hydroxy-5"'-benzylisouvarinol-B. Org Lett 7, 5609-5612.

Walker, J. E., Saraste, M., Runswick, M. J. \& Gay, N. J. (1982). Distantly related sequences in the alpha- and beta-subunits of ATP synthase, myosin, kinases and other ATP-requiring enzymes and a common nucleotide binding fold. EMBO J 1, 945-951.

White, E. L., Suling, W. J., Ross, L. J., Seitz, L. E. \& Reynolds, R. C. (2002). 2-Alkoxycarbonylaminopyridines: inhibitors of Mycobacterium tuberculosis FtsZ. J Antimicrob Chemother 50, 111-114. 